\title{
Absorption and emission spectroscopies of homogeneous and inhomogeneously broadened multilevel systems in strong light fields ${ }^{\text {a) }}$
}

\author{
Bernhard Dick ${ }^{\text {b) }}$ and Robin M. Hochstrasser \\ Department of Chemistry, University of Pennsylvania, Philadelphia, Pennsylvania 19104
}

(Received 15 March 1984; accepted 4 June 1984)

\begin{abstract}
A method is introduced to calculate for a model set of molecular levels the spectral line shapes expected for a variety of conventional laser experiments including absorption, hole burning, fluorescence line narrowing, and Raman scattering. The method allows the incident laser field to have arbitrary intensity. Furthermore, the effects of model Gaussian or Lorenzian inhomogeneous distributions are readily incorporated. Earlier results for a two-level system are easily obtained and new results are presented for inhomogeneously broadened two and three level systems, and for the effects of pure dephasing on the strong field spectra. In particular we have described the differences between fluorescence and Raman in strong fields, and the effect of strong fields on the spontaneous emission of inhomogeneously broadened transitions. Some predictions are made regarding line narrowing experiments in the strong-field limit.
\end{abstract}

\section{INTRODUCTION}

Many different types of experiments using lasers are carried out in which the result of the interactions with the radiation can be well modeled by two or three level molecular systems coupled to near resonant light fields. Most of the resulting dynamical and spectroscopic effects are already understood in principle, yet there is no standard approach to multilevel problems. Different authors use different approximations and assumptions; some descriptions are valid for only weak fields, others for processes in the absence of dephasing effects, and others for only homogeneous systems. Clearly there is a need for a unified approach to such problems, particularly aimed at being useful to spectroscopists who will require an explicit formulation of line shapes, line positions, and intensities corresponding to the various dynamical processes that can occur on excitation of sets of molecular energy levels in light fields of arbitrary strengths. In this paper we describe such a general approach which can be applied in a straightforward manner to a wide variety of experiments currently possible with lasers. We confine the discussion to incoherent spectroscopies such as optical absorption, fluorescence, resonance Raman, fluorescence line narrowing, optical hole burning, and variants on these standard methods. The approach involves correlation function techniques and yields the incoherent spectra in terms of the steady state density matrix elements that are frequently used to describe coherent optical phenomena. The nonperturbative method used for obtaining the density matrix has already proved useful in the discussion of strong field effects in nonlinear coherent spectroscopies. ${ }^{1}$

Besides the rotating wave approximation (RWA), the standard technique for calculating the density matrix in-

\footnotetext{
a) This research was supported by a PHS grant GM12592 and in part the work supported by the National Science Foundation, MRL Program, under Grant No. DMR-8216718.

b) A research Fellowship of the "Deutsche Forschungsgemeinschaft" is gratefully acknowledged. Permanent address: Max Planck Institut für Biophysikalische Chemie, Abt. Laserphysik, Am Fassberg, D 3400 Göttingen, Federal Republic of Germany.
}

volves two further approximations: First, Markovian relaxation dynamics are assumed which means the correlation times of the bath states are shorter than those of the system or the inverse Rabi frequencies of the field. ${ }^{2}$ This leads to exponential decay and Lorentzian line shapes in the weak field limit. Although the Markov approximation has proved very useful it cannot completely describe the processes responsible for the far wings of resonance lines. Methods to describe the relaxation more generally via higher order correlation functions have been developed and reviewed recently. ${ }^{3}$ In this paper we will retain the Markov approximation and the results should be applicable to gaseous systems of low pressure of condensed systems at low temperatures. The second approximation involves the perturbative treatment of the external driving fields leading to a hierarchical ordering of the optical nonlinear responses in powers of the field strengths. This approximation certainly breaks down when the field is resonant with a transition whose width is smaller than the Rabi frequency of the field, since the perturbation series diverges in this case. In the following we will refer to such a situation as a strong field interaction, and we are interested in nonperturbative solutions for the line shapes of such nonlinear spectra.

Formulas similar or equivalent to some of the results that we will give in the following sections have already been given by other authors using different methods. Mollow thoroughly investigated the two-level case, ${ }^{4-7}$ and some three level situations have been discussed by Mollow, ${ }^{8,9} \mathrm{Mu}$ kamel et al. ${ }^{10-12}$ Cohen-Tannoudji et al., ${ }^{13-15}$ Szöke and Courtens, ${ }^{16,17}$ Rabin et al. ${ }^{18,19}$ and others. Several papers were aimed at understanding the Raman and fluorescence line shapes in weak fields. ${ }^{20-25}$ We show here that in the multiple rotating reference frame all these situations can be described in a single, time independent notation. The calculations reduce to matrix inversions of dimension $2 \times 2$ or $4 \times 4$, with these matrices being subproblems of a $9 \times 9$ matrix given in our previous work. ${ }^{1}$ This approach also accounts for incoherent population transfer (feeding) effects not included in some of the above mentioned studies.

There are several contributions to transition linewidths, 
namely the homogeneous width, power broadening, and inhomogeneous widths. While the first two are accounted for using previous methods, the latter require an average of the corresponding quantity over the distribution function of the inhomogeous ensemble. Depending on the location of singularities of these characteristic quantities in the complex plane, the averaged spectrum can exhibit linewidths determined mainly by homogeneous relaxation parameters. Such so called line narrowing capabilities have been demonstrated for coherent nonlinear spectroscopies in the weak field limit such as CSRS and difference frequency generation. Here we extend the averaging method to strongly driven systems and study the predictions for incoherent spectroscopies that would correspond to fluorescence line narrowing and hole burning in the weak field limit.

Emission and absorption are usually described semiclassically from the work done by the polarization $P$ of the medium on an infinitesimal probe field $E_{s}$ :

$$
\Omega=\left\langle\frac{\partial \mathbf{P}}{\partial t} \mathbf{E}_{s}^{*}\right\rangle \text {. }
$$

The spectrum is found by evaluating Eq. (1.1) for each Fourier component:

$$
\Omega=\omega \operatorname{Im}\left\{P_{\omega} \cdot E_{\omega}^{*}\right\} .
$$

The macroscopic polarization is then calculated in terms of the density matrix. In this semiclassical picture one can describe spontaneous emission as being stimulated by the black body zero-photon field at $\omega_{s}{ }^{25}$ However this can give erroneous results when the lower molecular level of the transition is populated, i.e., the method predicts attenuation of the zero-photon field. A method that gives correct results both for spontaneous and stimulated processes employs correlation functions of molecular transition operators.

In Sec. II we show how such correlation functions can be evaluated in the multiple rotating frame approximation for arbitrary field strengths. Section III presents a general method to average these nonlinear spectra over Lorentzian or Gaussian inhomogenous distributions. Sections IV and V deal with applications to two- and three-level systems.

\section{CALCULATION OF CORRELATION FUNCTIONS IN THE FULLY RESONANT MULTILEVEL MODEL}

The spectrum of the emitted light under steady state conditions connected with the transition from an upper molecular level $|\mu\rangle$ to a lower level $|v\rangle$ is given by the Fourier transform of a correlation function $G(t)(6)$ :

$$
g(\omega)=\int_{-\infty}^{+\infty} d t e^{i \omega t} G(t) .
$$

For stimulated processes, this correlation function involves the commutator of the excitation and deexcitation operators $a_{\mu \nu}=|v\rangle\langle\mu|$ and $a_{\nu \mu}=a_{\mu \nu}^{*}$, while the spontaneous process is described by the emission time ordering only ${ }^{26}$ :

$$
\begin{aligned}
& G_{\mathrm{st}}(t)=\left\langle\left[a_{\nu \mu}, a_{\mu v}(t)\right]\right\rangle, \\
& G_{\mathrm{sp}}(t)=\left\langle a_{v \mu} a_{\mu \nu}(t)\right\rangle .
\end{aligned}
$$

The brackets in Eqs. (2.2) and (2.3) indicate averaging with the steady state density operator $\rho^{0}$, e.g.,

$$
\left\langle a_{\mu \nu}(t)\right\rangle=\operatorname{Tr}\left\{\rho^{0} a_{\mu v}(t)\right\}=\rho_{\mu v}(t) .
$$

While the spontaneous spectrum $g_{\text {sp }}$ is always positive, the stimulated spectrum $g_{\text {st }}$ can have both positive and negative values, the later corresponding to absorption rather than emission.

The $a_{\mu v}(t)$ are readily found using a method of $\operatorname{Lax}^{27,28}$ as applied by Mollow. ${ }^{4,8}$ Since the $a_{\mu \nu}$ form a complete operator basis for any time, we can make the expansion:

$$
a_{\mu v}(t)=\sum_{\sigma \tau} B_{\mu v, \sigma \tau}(t) a_{\sigma \tau}(0) \text {. }
$$

The coefficients $B_{\mu \nu, o r}$ are the matrix elements of the resolvent operator, ${ }^{29,30}$ a transformation operator in Liouville space, often called a tetradic matrix. ${ }^{3}$ It follows from $\mathrm{Eq}$. (2.5) that only the $\mu \nu$ row of this matrix is needed. This fact is responsible for the facile implementation of our multirotating frame method, as will be seen later. Once the coefficients $B_{\mu v, \sigma \tau}$ are found the correlation function is obtained by inserting Eq. (2.5) into Eqs. (2.2) and (2.3):

$$
\begin{aligned}
& G_{\mathrm{st}}(t)=\sum_{\sigma}\left\{B_{\mu v, \sigma v}(t) \rho_{\sigma \mu}^{0}-B_{\mu \nu, \mu \sigma}(t) \rho_{v \sigma}^{0}\right\}, \\
& G_{\mathrm{sp}}(t)=\sum_{\sigma} B_{\mu v, \sigma v}(t) \rho_{\sigma \mu}^{0} .
\end{aligned}
$$

Here we have used $a_{\nu \mu} a_{\sigma \tau}=a_{\sigma \mu} \delta_{v \tau}$ following from the definitions and the orthogonality of the molecular eigenfunctions. The steady state density matrix elements $\rho_{\mu \nu}^{0}$ must be evaluated in the absence of the signal field in both the spontaneous and the stimulated case. It is often easier to find the Laplace transform of the coefficients $B^{31}$ :

$$
B^{L}(s)=\int_{0}^{\infty} e^{-s t} B(t) d t
$$

Replacing $B(t)$ with $B^{L}(s)$ in Eq. (2.6) will directly give the Laplace transform $g^{L}(s)$ of $g(t)$. The Fourier transform and consequently the spectrum can be found without backtransforming to the time domain by

$$
g(\omega)=2 \operatorname{Re}\left\{g^{L}(-i \omega)\right\}
$$

which is a consequence of the relation $G(-t)=G^{*}(t)$.

To find the coefficients of the $B$ matrix we take the average of Eq. (2.5) according to Eq. (2.4):

$$
\rho_{\mu \nu}(t)=\sum_{\sigma \tau} B_{\mu \nu, \sigma \tau}(t) \rho_{\sigma \tau}^{o} .
$$

The required coefficients are, therefore, the coefficients of the solution of the Liouville equation for the density operator. In our previous paper ${ }^{1}$ we solved the Liouville equation in Laplace space:

$$
\tilde{\rho}^{L}(s)=\tilde{B}^{L}(s) \cdot \rho^{0}
$$

where $\tilde{\rho}=U_{\rho}$ is the transformed density matrix in the rotating frame, and $\tilde{B}(s)$ is the inverse of the Liouville superoperator $(X-s 1)$ in that frame. The transformation superoperator $U$ has the matrix elements:

$$
U_{\mu \nu, \sigma \tau}=\delta_{\mu \sigma} \delta_{v \tau} \exp \left(i s_{\mu \nu} \omega_{j} t\right)
$$

where $\omega_{j}$ is the (positive) transformation frequency associated with the $\mu v$ level pair, and $s_{\mu v}$ is the sign of $\omega_{\mu v}=\left(E_{\mu}-E_{v}\right) / \hbar .^{32}$ The relation between Eq. $(2.10)$ and its counterpart in the fixed frame is found by application of 
the transformation (2.11) to Eq. (2.9) followed by Laplace transformation. The result is

$$
B_{\mu \nu, \sigma \tau}^{L}(s)=\tilde{B}_{\mu v, \sigma \tau}^{L}\left(s+i s_{\mu \nu} \omega_{j}\right) .
$$

This means, that for each row of the $\tilde{B}^{L}$ matrix in the rotating frame the argument is shifted by the same constant. For the spectrum [Eq. (2.8)] we need the coefficients $B^{L}\left(-i \omega_{j}\right)$, or $\tilde{B}^{L}\left[i\left(\omega_{j}-\omega_{s}\right)\right]=\tilde{B}^{L}(i v) .{ }^{33}$ The final expressions for the spectra evaluated in the rotating frame are

$$
\begin{gathered}
g_{\mathrm{st}}\left(\omega_{s}\right)=2 \operatorname{Re}\left\{\sum_{\sigma}\left(\tilde{B}_{\mu v, \sigma v}^{L}(i v) \rho_{\sigma \mu}^{0}-\tilde{B}_{\mu \nu, \mu \sigma}^{L}(i v) \rho_{v \sigma}^{0}\right)\right\}, \\
\rho_{\mathrm{sp}}\left(\omega_{s}\right)=2 \operatorname{Re}\left\{\sum_{\sigma} \tilde{B}_{\mu v, \sigma v}^{L}(i v) \rho_{\sigma \mu}^{0}\right\} .
\end{gathered}
$$

If $\omega_{j}$ is not already defined by the ingoing field frequencies, we may identify it with $\omega_{s}$, i.e., evaluate $\tilde{B}^{L}(0)$. This is the situation in all of the three-level cases discussed later. In the two-level case $\omega_{j}$ is assigned to $\omega_{L}$, and consequently $v=\omega_{L}-\omega_{s}$.

\section{AVERAGE OVER INHOMOGENOUS DISTRIBUTIONS}

For an inhomogeneously broadened system modeled by a static distribution of energies the density matrix must be averaged over the corresponding distribution function. The averaged density matrix may then be used to calculate coherent as well as incoherent spectra of the inhomogneously broadened system. This will account for hole burning and fluorescence line narrowing in the incoherent case, and for line narrowing effects in coherent spectroscopies such as CSRS or difference frequency generation. ${ }^{34}$ Below we give a general method to calculate such averages for systems subject to light fields of arbitrary strength using two types of model distribution functions, namely Lorentzians and Gaussians. In the limit of weak light fields the expressions reduce to those known from the perturbative treatment of the fields. The distribution function is in general $(N-1)$ dimensional for an $N$ level system, but can be reduced to a one dimensional problem under the assumption that all energy shifts are correlated. This is valid in the case of a Doppler broadened system, but not necessarily in glasses or crystals. $^{35}$ The two-dimensional problem for the three-level system could be solved by a two-step averging procedure similar to the one discussed in Ref. 34 . This would require a numerical integration in the second step for Gaussian model functions.

As will be seen later the functions to be averaged are always of the form:

$$
f(x)=h(x) / g(x)
$$

where $x$ is the inhomogeneous distribution parameter and $h$ and $g$ are polynomials with no common linear factors. In all cases to be encountered $g$ is of higher order in $x$ than $h$, and all $n$ zeroes $p_{j}$ of $g$ are different. Thus the function $f(x)$ can be expanded in partial fractions around these first order poles:

$$
f(x)=\sum_{j=1}^{n} c_{j}\left(x-p_{j}\right)^{-1}
$$

The coefficients are found via

$$
c_{j}=h\left(p_{j}\right) \prod_{k \neq j}\left(p_{j}-p_{k}\right)^{-1}
$$

which is easily adapted to numerical evaluation. Averaging the expression (3.2) over a Lorentzian distribution with width $2 b$ yields

$$
\langle f\rangle_{L}=-\sum_{j=1}^{n} c_{j} s_{j}\left(i b+s_{j} p_{j}\right)^{-1},
$$

where $s_{j}=\operatorname{sign}\left[\operatorname{Im}\left(p_{J}\right)\right]$. The average over a Gaussian with variance $b$ yields

$$
\langle f\rangle_{G}=i \sqrt{\pi / 2} b^{-1} \sum_{j=1}^{n} c_{j} s_{j} \omega\left(s_{j} p_{j} / \sqrt{2 b}\right) .
$$

Here $w$ denotes the complex error function and $s_{j}$ is defined as above. For numerical evaluation, tables of $w$ as well as sufficiently accurate approximation formulas are available. ${ }^{36}$ Thus the inhomogeneous averaging is reduced to finding the poles of $f(x)$, i.e., the roots of $g(x)$. In the applications dealt with in this paper, as well as in all other situations involving only one strong field, the denominator polynomial $g(x)$ is a product of terms quadratic in $x$. Therefore all the poles can be found analytically.

\section{THE TWO-LEVEL SYSTEM}

The line shape functions for absorption and emission in a homogeneous two-level system are well known from Mollow's work ${ }^{5,6}$ and have been discussed frequently in different theoretical frameworks. We briefly rederive these spectral functions to demonstrate our time independent method and pass then to the discussion of inhomogeneous ensembles. In accordance with the nomenclature of our previous work ${ }^{1}$ we label the upper level $|b\rangle$ and the lower level $|a\rangle$ as schematically shown in Fig. 1(a). An external light field with frequency $\omega_{L}$ and Rabi frequency $W=\mu_{a b} \epsilon_{L}$ is driving the system close to resonance, and the signal field frequency $\omega_{s}$ is also

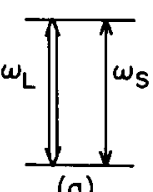

(a)

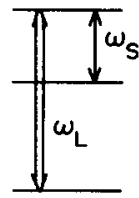

(b)

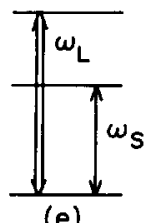

(e)

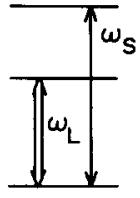

(c)

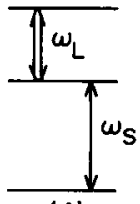

(f)

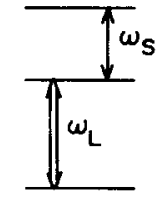

(d)

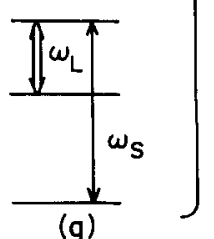

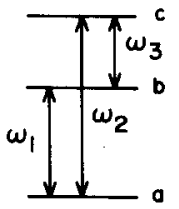

FIG. 1. Possible combinations of driving and signal fields in fully resonant two-level and three-level systems. The right-hand side shows the numbering convention for the transformation frequencies $\omega_{\boldsymbol{i}}$. For example, to calculate case (b), $\omega_{3}$ is identified with $\omega_{s}$, and $\omega_{1}$ with $\omega_{L}-\omega_{s}$. 
close to resonance. The Liouville equation of motion for the excitation operators $\tilde{a}_{\mu \nu}$ in the rotating frame and in the absence of the signal field is [see Eq. (31) of Ref. 1]:

$$
\begin{gathered}
{\left[\begin{array}{cccc}
S & -\Gamma_{b b} & i W^{*} & -i W \\
0 & \Gamma_{b b}+s & -i W^{*} & i W \\
i W & -i W & i \Delta_{a b}+s & 0 \\
-i W^{*} & i W^{*} & 0 & -i \Delta_{a b}^{*}+s
\end{array}\right]\left[\begin{array}{l}
\tilde{a}_{a a}(s) \\
\tilde{a}_{b b}(s) \\
\tilde{a}_{a b}(s) \\
\tilde{a}_{b a}(s)
\end{array}\right]} \\
=\left[\begin{array}{l}
\tilde{a}_{a a}^{0} \\
\tilde{a}_{b b}^{0} \\
\tilde{a}_{a b}^{0} \\
\tilde{a}_{b a}^{0}
\end{array}\right] .
\end{gathered}
$$

Here $\Delta_{a b}=\omega_{a b}+\omega_{L}-i \Gamma_{a b}$ with $\Gamma_{a b}$ and $\Gamma_{b b}$ being the inverse $T_{2}$ and $T_{1}$ relaxation parameters, respectively. Inversion of this $4 \times 4$ Liouvillian matrix gives the required $\tilde{B}^{L}$ coefficients, of which only the following three are necessary:

$$
\begin{aligned}
& \tilde{B}_{b a, a a}^{L}(s)=-W\left(\Gamma_{b b}+s\right)\left(\Delta_{a b}-i s\right) /[s \cdot D(s)], \\
& \tilde{B}_{b a, b b}^{L}(s)=-W\left(\Gamma_{b b}-s\right)\left(\Delta_{a b}-i s\right) / \cdot[s \cdot D(s)], \\
& \tilde{B}_{b a, b a}^{L}(s)=\left[2 W^{2}+i\left(\Gamma_{b b}+s\right)\left(\Delta_{a b}-i s\right)\right] / D(s),
\end{aligned}
$$

with

$$
D(s)=\left(\Gamma_{b b} s\right)\left(\Delta_{a b}-i s\right)\left(\Delta_{a b}^{*}+i s\right)+4 W^{2}\left(\Gamma_{a b}+s\right) .
$$

The steady state density matrix is

$$
\begin{aligned}
& \rho_{a c}^{0}=\left(\Gamma_{b b} \Delta_{a b} \Delta_{a b}^{*}-2 W^{2} \Gamma_{a b}\right) / D(0), \\
& \rho_{b b}^{0}=2 W^{2} \Gamma_{a b} / D(0), \\
& \rho_{a b}^{0}=-W \Gamma_{b b} \Delta_{a b}^{*} / D(0) .
\end{aligned}
$$

With these coefficients set into Eq. (2.13) one obtains directly the expressions for the spontaneous and stimulated emission and absorption line shapes:

$$
\begin{aligned}
g_{s t}\left(\omega_{s}\right)= & 2 \operatorname{Re}\left\{\left[\left(\Delta_{a b}+v\right)\left(v-i \Gamma_{b b}\right) \Gamma_{b b} \Delta_{a b} \Delta_{a b}^{*}+2 W^{2} \Gamma_{b b}\right.\right. \\
& \left.\left.\times \Delta_{a b}^{*} 2\right] /[D(0) \cdot D(s)],\right\}
\end{aligned}
$$

$$
\begin{aligned}
g_{s p}\left(\omega_{s}\right)= & 2 \operatorname{Re}\left\{\left[\left(\Gamma_{b b}+i v\right)\left(\Delta_{a b}+v\right)\left(\Gamma_{b b} \Delta_{a b}^{*}-2 \Gamma_{a b} v\right)\right.\right. \\
& \left.\left.+4 i v \Gamma_{a b} W\right] /[D(0) D(i v) \cdot(i v+\epsilon)]\right\} .
\end{aligned}
$$

Equations (4.4) give the exact (RWA) result for a two-level system interacting with a light field of arbitrary strength. ${ }^{37}$

To perform the average over an inhomogeneously broadened ensemble, $\Delta_{a b}$ is replaced by $\Delta_{a b}+x$, where $\omega_{a b}$ now refers to the center frequency of the inhomogeneous distribution $\omega_{a b}^{0}$. The poles needed for the partial fraction expansion are then found as the zeros of $D(i v)$ and $D(0)$. This yields

$$
\begin{gathered}
D(i v)=\left(\Gamma_{b b}+i v\right)\left(\Delta_{a b}+v+x\right)\left(\Delta_{a b}^{*}-v+x\right) \\
\quad+4 W^{2}\left(\Gamma_{a b}+i v\right)=0 \\
p_{1,2}=-\omega_{a b}^{0}-\omega_{L} \pm\left\{\left(v-i \Gamma_{a b}\right)^{2}-4 W^{2}\left(\Gamma_{a b}+i v\right) /\right. \\
\left.\left(\Gamma_{b b}+i v\right)\right\}^{1 / 2} \\
p_{3,4}=-\omega_{a b}^{0}-\omega_{L} \pm i \Gamma_{a b} \cdot\left(1+4 W^{2} / \Gamma_{a b} \Gamma_{b b}\right)^{1 / 2}
\end{gathered}
$$

\section{A. Spontaneous spectra: Fluorescence and Raylolgh scattering}

Figures 2-6 show various line shape characteristics for spontaneous processes in two-level systems. When there is no pure dephasing, i.e., $\Gamma_{b b}=2 \Gamma_{a b}$, spectral line shapes for increasing strength of the driving light field are given in Fig. 2 with the driving field on resonance. In the weak field limit only a sharp Rayleigh scattering spike is present, which with increasing field strength broadens and developes two sidebands at $\omega_{L} \pm 2 W$. When the pump beam is detuned from resonance (Fig. 3 ) the spectrum remains symmetric with respect to the pump frequency, but the intensity ratio of the center band to the sidebands may vary considerably. In particular the sidebands can become more intense than the center band. The introduction of pure dephasing drastically changes this picture. Even in the weak field limit the emission line is broad with width $\Gamma_{a b}$ and centered at $\omega_{a b}^{0}$ not at $\omega_{L}$ (Fig. 4). This is the expected behavior of fluorescence rather than Rayleigh scattering. With increasing pump field strength again a three line pattern evolves, but the intensities are no longer symmetric, the lines closer to $\omega_{a b}$ being enhanced with respect to the others. In the strong field limit the center peak moves to $\omega_{L}$ as would be typical for a Ramantype scattered spectrum. Figures 5 and 6 show the spectra stimulated for a broad inhomogeneous distribution of Gaussian shape without and with pure dephasing, respectively. The spectra are very similar to the spectra of the corresponding homogeneous systems. Detuning the driving field from the center of the inhomogeneous distribution has however, only the effect of shifting the whole spectrum as long as the detuning is smaller than the inhomogeneous width. In summary, the fluorescence spectrum is found to be line narrowed even in the case of strong driving light fields. From the intensity ratio, widths, and separations of the resulting three band spectra the molecular parameters $\Gamma_{a b}, \Gamma_{a b}^{\prime}$ (the pure dephasing contribution), and $\mu_{a b}$ could be extracted.

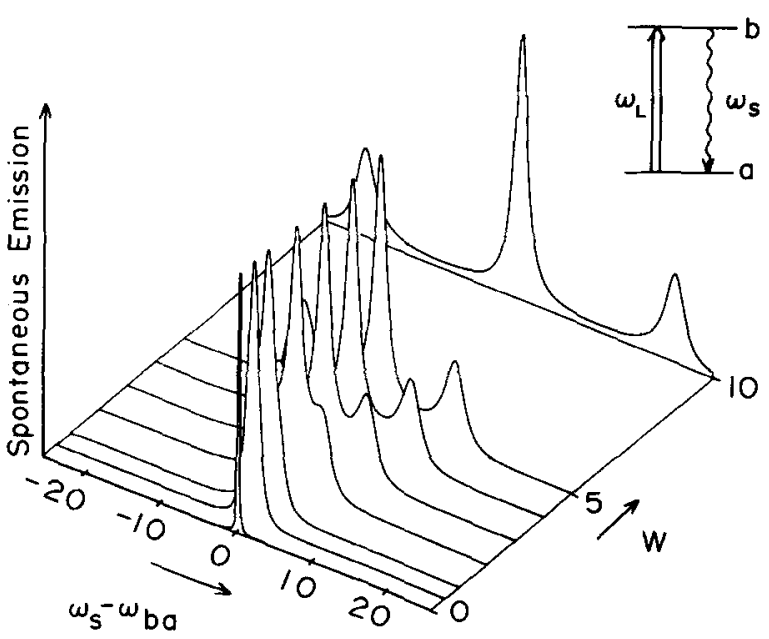

FIG. 2. Line shape simulation for the spontaneous emission spectra in a strongly driven two-level system. Parameters are $\Gamma_{a b}=1, \Gamma_{b b}=2$ (no pure dephasing) and $d=\omega_{L}-\omega_{b a}=0$ (no detuning of the pump field). $W$ is the Rabi frequency of the driving field. All lines are normalized to their maximum height, but the elastic Rayleigh peak cutoff. (A value of $10^{-4}$ was used for $\epsilon$, for details see the text.) 


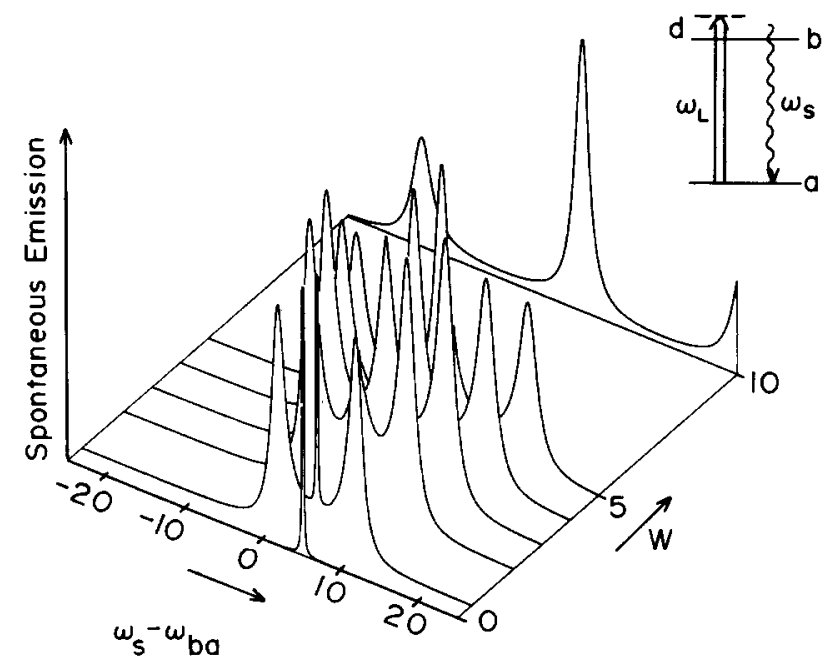

FIG. 3. Same as Fig. 2, but with the pump detuned by $d=5$ (Ref. 45).

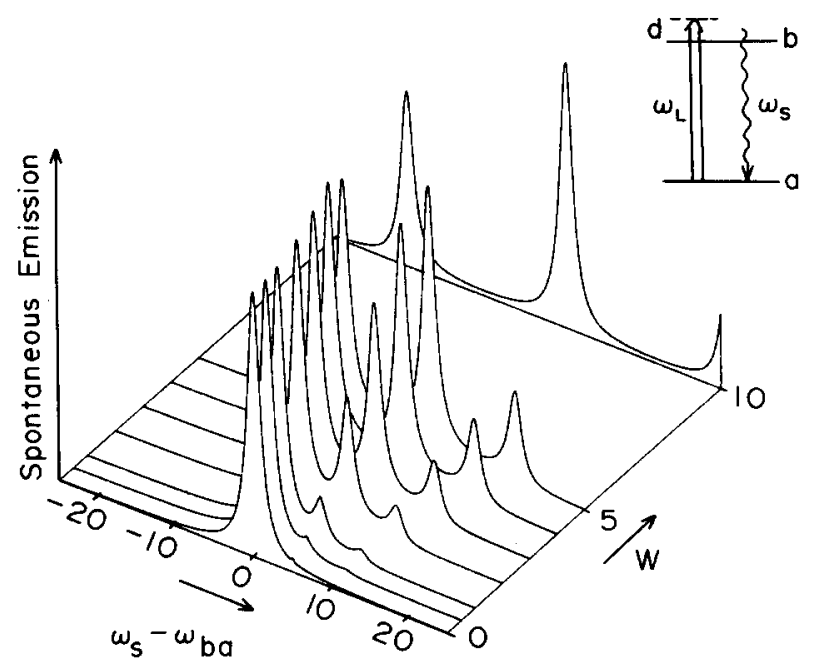

FIG. 4. Same as Fig. 3, but with $\Gamma_{b b}=1$. $\Gamma_{a b}$ now contains $50 \%$ pure dephasing.

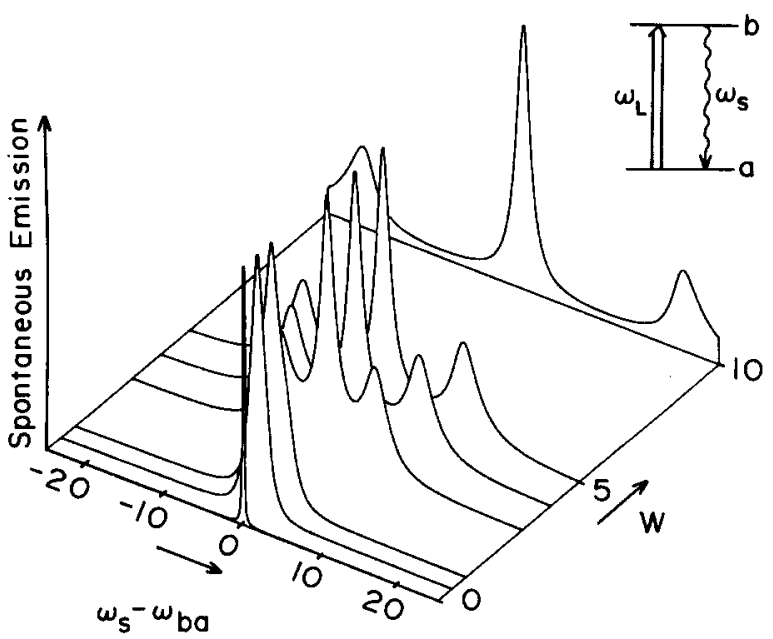

FIG. 5. Same as Fig. 2, but for a Gaussian inhomogeneous distribution with $\sigma=\mathbf{5 0}$.

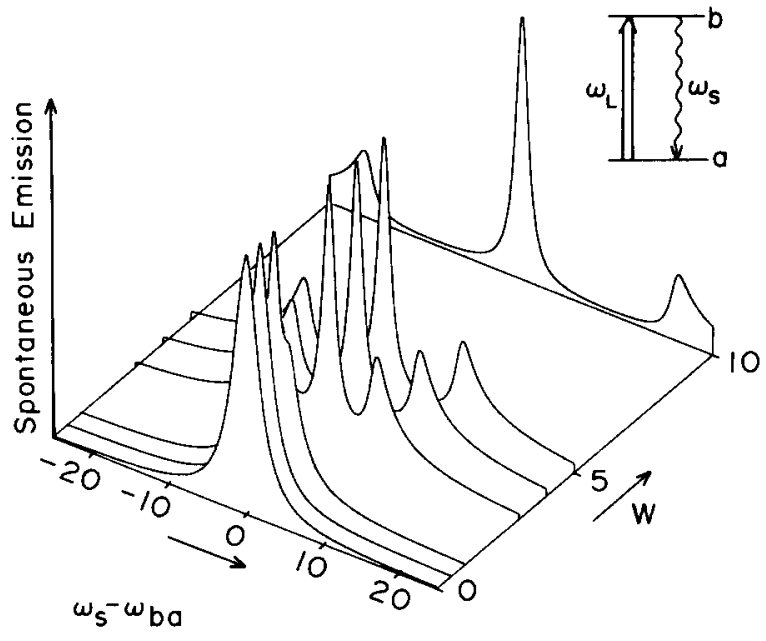

FIG. 6. Same as Fig. 5, but with $\Gamma_{b b}=1$.

\section{B. Absorption and stimulated emission}

The spectra for the stimulated process behave quite differently to field variations. In Fig. 7 the absorption spectrum is shown for the same conditions used in Fig. 2 for the spontaneous case: No pure dephasing, on resonance excitation, and no inhomogeneous distribution. For weak driving field the conventional Lorentzian absorption line is produced, which broadens and splits for increasing field. For even higher fields "negative" peaks develop between these two absorption lines. These describe amplification of the signal wave and, consequently, stimulated emission takes place at these points in the spectrum. With the pump frequency detuned from resonance the spectrum becomes unsymmetric as shown in Fig. 8. In the region closer to the $\omega_{a b}$ resonance the absorption is enhanced, while at the opposite end of the spectrum the emission peak builds up. The spectra of both detuned and on-resonance driven systems remain essentially unaltered when pure dephasing is introduced, in sharp contrast with the fluorescence case. Spectra for the inhomogeneously averaged are given in Fig. 9. They are not influenced

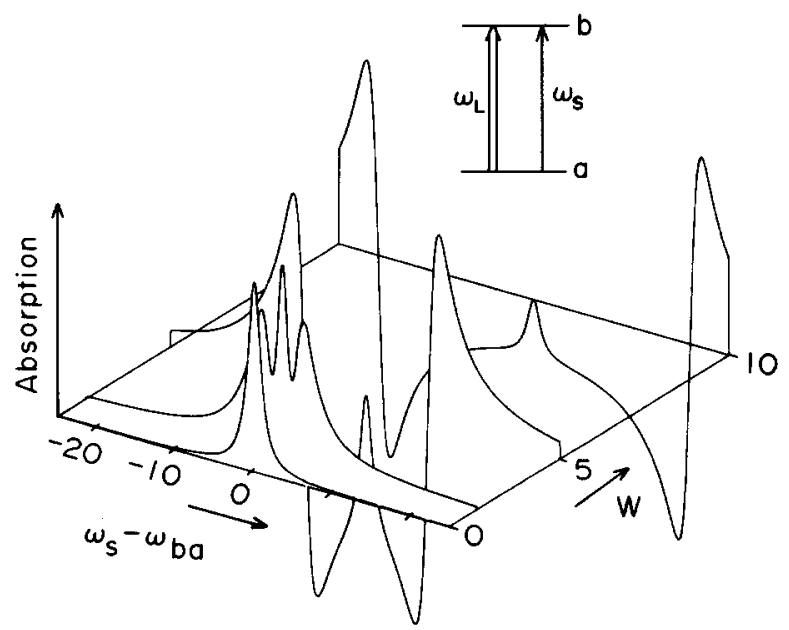

FIG. 7. Absorption spectrum for a strongly driven two-level system. $\mathbf{P a}$ rameters are the same as in Fig. 2: $\Gamma_{a b}=1, \Gamma_{b b}=2$, and $d=0$. 


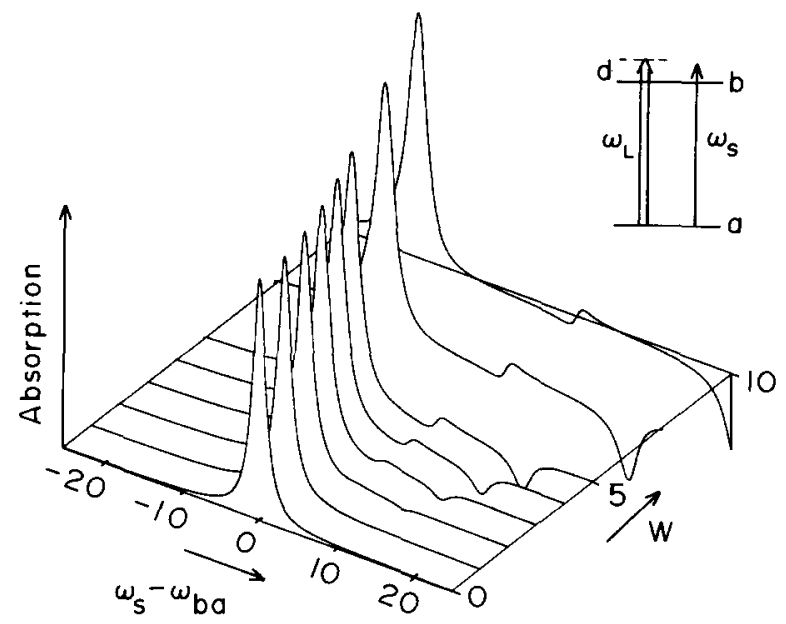

FIG. 8. Same as Fig. 7, but with the driving field detuned by $d=5$.

by detuning or the presence of pure dephasing. For $W=0$ the absorption reflects the broad inhomogeneous band. Small values of $W$ will burn a Lorentzian hole in this shape which will broaden and split with increasing field strength. The sample will become essentially transparent in the region $\omega_{L}-2 W$ to $\omega_{L}+2 W$, since the emission and absorption contributions of different subsets of the inhomogeneous distribution cancel in this range. No net stimulated emission is expected according to this analysis.

\section{THREE LEVEL SYSTEMS}

All three level problems reduce to the inversion of a $2 \times 2$ matrix that couples $a_{\mu \nu}$ via the strong field to another transition operator. Six cases arise depending on whether the lower or the upper level of the transition $\mu \rightarrow v$ couples to the strong light field, and whether the third level lies below, between or above the emitting/absorbing level pair $\mu v$. All these $2 \times 2$ problems are easily found as submatrices of the full Liouvillian matrix for the three level system, which is given in Eq. (34) of Ref. 1. Figure 1 gives a schematic repre-

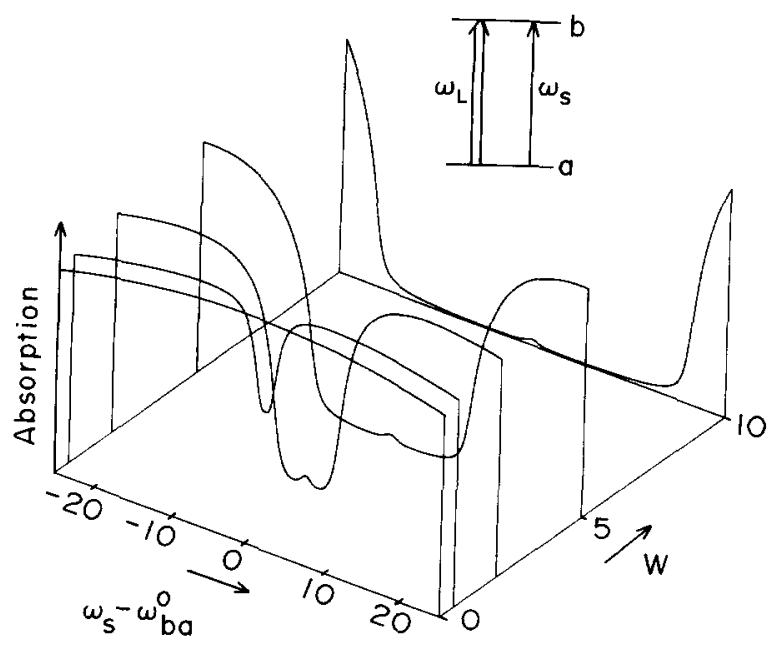

FIG. 9. Absorption spectrum of a strongly driven two-level system having a Gaussian inhomogeneous distribution. Parameters are: $\Gamma_{a b}=1, \Gamma_{b b}=1$, $d=0$, and $\sigma=50$. sentation of these six situations. In the following we will discuss two of these in detail.

\section{A. Fluorescence and resonance Raman spectra}

The level system under consideration is pictured in Fig. 1(b). The strong light field drives the $a c$ level pair and emission is observed near resonant with the $c b$ transition. The $2 \times 2$ problem takes the explicit form:

$$
i\left(\begin{array}{cc}
\Delta_{b c}^{*} & W^{*} \\
W & -\Delta_{a b}
\end{array}\right)\left(\begin{array}{c}
\tilde{a}_{c b} \\
\tilde{a}_{a b}
\end{array}\right)=\left(\begin{array}{c}
\tilde{a}_{c b}^{0} \\
\tilde{a}_{a b}^{0}
\end{array}\right) .
$$

From the inverted matrix $\tilde{B}^{L}$ we need two elements, namely,

$$
\begin{aligned}
& \tilde{B}_{c b, c b}=i \Delta_{a b}\left(\Delta_{a b} \Delta_{b c}^{*}+W^{2}\right)^{-1}, \\
& \tilde{B}_{c b, a b}=i W^{*}\left(\Delta_{a b} \Delta_{b c}^{*}+W^{2}\right)^{-1} .
\end{aligned}
$$

The steady state density matrix has been given in our previous work ${ }^{1}$ in Eq. 48 :

$$
\begin{aligned}
& \rho_{c c}^{0}=2 W^{2} \Gamma_{a c} / \Gamma_{c c} D_{2}, \\
& \rho_{b b}^{0}=2 W^{2} \Gamma_{a c} \gamma_{c b} / \Gamma_{b b} \Gamma_{c c} D_{2}, \\
& \rho_{a c}^{0}=-W \Delta_{a c}^{*} / D_{2}, \\
& D_{2}=\Delta_{a c} \Delta_{a c}^{*}+q W^{2}, \\
& q=2 \Gamma_{a c}\left(2 \Gamma_{b b}+\gamma_{c b}\right) / \Gamma_{b b} \Gamma_{c c} .
\end{aligned}
$$

Setting Eqs. (5.2) and (5.3) into (2.13) yields for the spontaneous emission spectrum:

$$
\begin{aligned}
q_{\mathrm{sp}}\left(\omega_{s}\right)= & 2 W^{2} \cdot \operatorname{Im}\left\{\left(\Delta_{a c}^{*}-2 \Delta_{a b} / \Gamma_{c c}\right) /\right. \\
& \left.\left(\Delta_{a b} \Delta_{b c}^{*}+W^{2}\right)\right\} / D_{2} .
\end{aligned}
$$

Equation (5.4) can be written in the form:

$$
q_{\mathrm{sp}}\left(\omega_{s}\right)=2 W^{2} \cdot \operatorname{Im}\left\{\chi_{R R S}+\chi_{F}\right\}
$$

with the definitions:

$$
\begin{aligned}
& \chi_{R R S}=\Delta_{a c}^{*} D_{2}^{-1}\left(\Delta_{a b} \Delta_{b c}^{*}+W^{2}\right)^{-1}, \\
& \chi_{F}=-2 \Delta_{a b} \Gamma_{a c} \Gamma_{c c}^{-1} D_{2}^{-1}\left(\Delta_{a b} \Delta_{b c}^{*}+W^{2}\right)^{-1}
\end{aligned}
$$

Expressed in these quantities, the stimulated emission spectrum has the form:

$$
q_{\mathrm{st}}\left(\omega_{s}\right)=2 W^{2} \operatorname{Im}\left\{\chi_{R R S}+\chi_{F}\left(1-\gamma_{c b} / \Gamma_{b b}\right)\right\},
$$

where $\gamma_{c b}$ is the rate of population feeding from state $|c\rangle$ to $|b\rangle$. The subscripts $R R S$ and $F$ are intended to denote the resonance Raman and fluorescence contribution. The usefulness of this partitioning is seen directly on expanding $\chi_{R R S}$ and $\chi_{F}$ in powers of $W^{2}$. The lowest order terms are

$$
\begin{aligned}
& \chi_{R R S}^{0}=\left(\Delta_{a c} \Delta_{a b} \Delta_{b c}^{*}\right)^{-1}, \\
& \chi_{F}^{0}=-2 \Gamma_{a c} \Gamma_{c c}^{-1}\left(\Delta_{a c} \Delta_{a c}^{*} \Delta_{b c}^{*}\right)^{-1} .
\end{aligned}
$$

These are the same results that Shen obtained via a perturbation expression of the density matrix to third order in the applied and generated fields ${ }^{25}$ using the semiclassical formula (1.2). It seems natural to transfer his identification of the $R R S$ and $F$ parts to the corresponding terms with arbitrary field strength in Eq. (5.6). The $R R S$, thus defined, originates from the coherence $\rho_{a c}^{0}$ multiplied by $B_{c b, a b}$, while the fluorescence part is determined by the population $\rho_{c c}^{0}$ for the spontaneous emission and the population difference $\rho_{c c}^{0}-\rho_{b b}^{0}$ for the stimulated emission, multiplied by $B_{c b, c b}$.

Before discussing line shapes we briefly address the in- 
homogeneous averaging procedure for the three-level system. The spectral function for a subset of the inhomogeneous distribution characterized by the parameter $x$ is found by replacing:

$$
\begin{aligned}
& \Delta_{a b} \rightarrow \Delta_{a b}+x, \\
& \Delta_{a c} \rightarrow \Delta_{a c}+\alpha x, \\
& \Delta_{b c} \rightarrow \Delta_{b c}+\beta x,
\end{aligned}
$$

where $\beta=\alpha-1$. This is a fully correlated model for the distribution. All denominators in all the six possible cases will be of the general form: $\left(\Delta_{1}+U x\right)\left(\Delta_{2}+V x\right)+A$. Consequently the poles are found directly as

$$
\begin{aligned}
P_{1,2}= & -(2 u v)^{-1} \\
& \times\left\{v \Delta_{1}+u \Delta_{2} \pm\left[\left(v \Delta_{1}-u \Delta_{2}\right)^{2}-4 u v A\right]^{1 / 2}\right\} .
\end{aligned}
$$

Line shapes for various values of the system parameters are simulated in Figs. 10-12. In all cases the ingoing field frequency was set detuned from the $\omega_{c a}$ center frequency by an amount $d=\omega_{L}-\omega_{c a}$ since this allows for spectral distinction of Raman and fluorescence contributions in certain situations. With no inhomogeneous distribution present the weak field limit [Eq. (5.8)] predicts a fluorescence line with width $\Gamma_{b c}$ peaked at $\omega_{s}=\omega_{c b}$ and a Raman line at $\omega_{s}=\omega_{L}-\omega_{b a}=\omega_{c b}+d$ and with width $\Gamma_{a b}$. The Raman term also contains the $\Gamma_{b c}$ resonance at $\omega_{c b}$ but with negative sign factor. In the absence of pure dephasing both these $\Gamma_{b c}$ resonances in $F$ and $R R S$ cancel out and only the $\Gamma_{a b}$ Raman resonance is seen:

$$
\begin{aligned}
\chi_{R R S}^{0}+\chi_{F}^{0}= & \left(\Delta_{a c} \Delta_{a c}^{*} \Delta_{a b}\right)^{-1} \\
& \times\left\{1+i\left(\Gamma_{b c}^{\prime}-\Gamma_{a c}^{i}-\Gamma_{a b}^{\prime}\right) / \Delta_{b c}^{*}\right. \\
& \left.-2 \Gamma_{a c}^{\prime} \Delta_{a b} / \Gamma_{c c} \Delta_{b c}^{*}\right\} .
\end{aligned}
$$

In other words, a peak at the fluorescence resonance frequency is dependent on the existence of pure dephasing only if the perturbation expansion is terminated at third order.

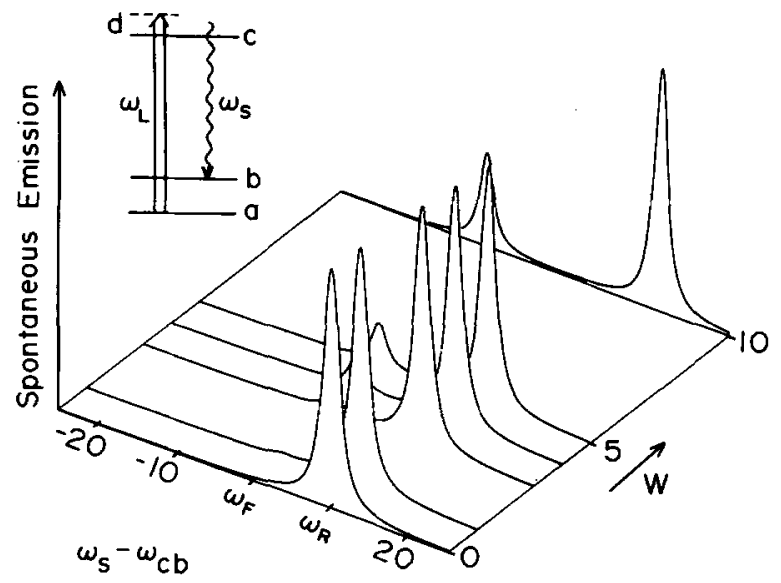

FIG. 10. Spontaneous emission spectra of the $|c\rangle \rightarrow|b\rangle$ transition in a three level system. The strong field pumps the $|a\rangle \rightarrow|c\rangle$ transition with a detuning $d=\omega_{L}-\omega_{c a}=10$. Linewidth parameters are $\Gamma_{a b}=1, \Gamma_{a c}=0.001$, $\Gamma_{b c}=1.001, \Gamma_{b b}=2, \Gamma_{c c}=0.002$ corresponding to the absence of pure dephasing. A small Gaussian inhomogeneous distribution with $\sigma=0.05$ and $\alpha=2$ is assumed. The frequencies $\omega_{s}=\omega_{c b}^{0}$ and $\omega_{s}=\omega_{L}-\omega_{b c}^{0}$ are marked as $\omega_{F}$ (fluorescence) and $\omega_{R}$ (Raman).

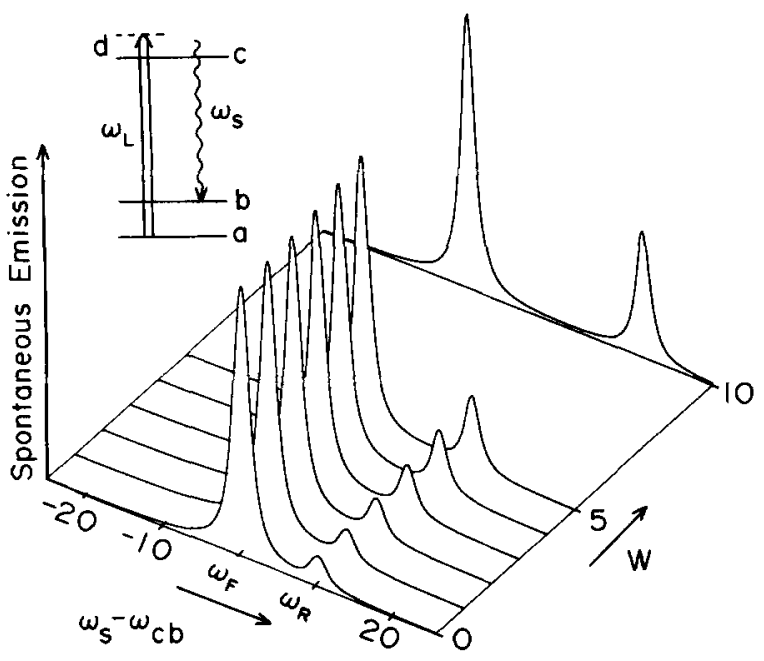

FIG. 11. Same as Fig. 10, but with a pure dephasing contribution $\Gamma^{\prime}=0.01$ added to all three linewidth parameters.

This corresponds to the case $W \rightarrow 0$ simulated in Fig. 10 . With increasing $W$ our nonperturbative treatment generates a second resonance which originates in the frequency region where the fluorescence peak is expected. These two resonances drift apart from each other, nearly linearly with increasing $W$. The linewidths of both lines are mixtures of $\Gamma_{a b}$ and $\Gamma_{a c}$. Loosely speaking the new resonance originating at $\omega_{s}=\omega_{c b}$ could be called "power induced fluorescence" in analogy to the "pure dephasing induced fluorescence" discussed above. Comparable effects of high laser power on the coherent generation of light were recently discussed by a number of authors. $1,38-43$

The effect of pure dephasing is demonstrated in Fig. 11. With the pure dephasing parameter equal for all level pairs and only $1 \%$ of the $\Gamma_{a b}$ and $\Gamma_{b c}$ parameters, the fluorescence at $\omega_{s}=\omega_{c b}$ becomes much stronger than the Raman scattering in contrast to the situation to the situation described in Fig. 10. Increasing the Rabi frequency of the driving field again shifts these resonances apart, but now it is the Raman peak that grows in relative intensity.

Figure 12 gives the result expected for a broad Gaussian inhomogeneous distribution. When the inhomogeneous

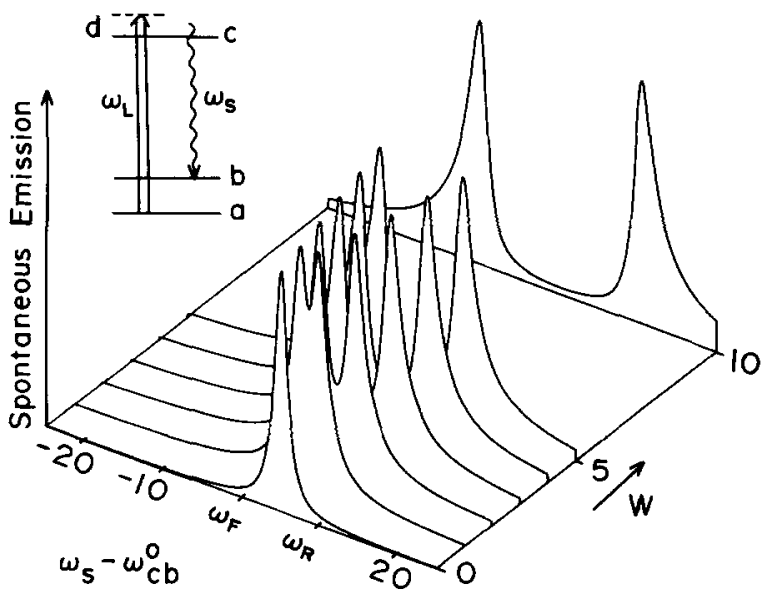

FIG. 12. Same as Fig. 10, but with the Gaussian inhomogeneous distribution broadened to $\sigma=50$. 
width is much larger than all homogeneous width parameters, the ensemble average of the perturbative result [Eq. (5.8)] predicts a single resonance at $\omega_{s}-\omega_{c b}=(\alpha-1) d / \alpha$. The width is a weighted average of the fluorescence $\left(\Gamma_{c b}\right)$ and $\operatorname{Raman}\left(\Gamma_{a b}\right)$ widths with the correlation factor $\alpha$ as scaling factor. For $\alpha=2$ the line falls exactly between the expected positions for Raman scattering and fluorescence. With increasing strength of the driving light field this line broadens and finally splits into two unsymmetrical lines with broad wings. Nearly the same pattern is obtained with or without pure dephasing. The emission in this case cannot always be specified as either fluorescence line narrowing or line narrowed resonance Raman scattering: For vanishing pure dephasing only Raman type terms contribute to the ensemble average, however with pure dephasing either the fluorescence or Raman contributions could dominate the spectrum of most subsets. In general the linewidth is a function of both the fluorescence and Raman widths. Analytic expressions for the line narrowed widths can be found performing the average with a Lorentzian distribution. ${ }^{34}$ In the limit of weak field and no pure dephasing, a linewidth for the emission line of

$$
\Gamma=\Gamma_{a b}+\Gamma_{a c} / \alpha
$$

is expected when $\alpha>0$. The line position is $\omega_{s}=\omega_{L}-\omega_{b a}-\left(\omega_{L}-\omega_{c a}\right) / \alpha$. In the presence of pure dephasing additional contributions arise at the same frequency but having widths:

$$
\Gamma=\Gamma_{b c}+(1-\alpha) \Gamma_{a c} / \alpha .
$$

The total line contains several terms and also cross products, but the sharpest contribution is expected to dominate the spectrum. In our example $\Gamma_{a b} \approx \Gamma_{b c}>\Gamma_{a c}$, so that the spectra have very nearly the homogeneous Raman and fluorescence linewidths.

\section{B. Saturation-type hole burning (non-photochemical)}

We consider now the situation in which the $a b$ level pair is driven by a strong light field and the $a \leftrightarrow c c$ transition spectrum is probed. [Fig. $1(\mathrm{c})$ ]. The two coefficients $\tilde{B}_{c a, c a}^{L}$ and $\tilde{B}_{c a, c b}^{L}$ are found by solving the equation:

$$
i\left(\begin{array}{cc}
\Delta_{a c}^{*} & -W^{*} \\
-W & \Delta_{b c}^{*}
\end{array}\right)\left(\begin{array}{l}
\tilde{a}_{c a} \\
\tilde{a}_{c b}
\end{array}\right)=\left(\begin{array}{c}
\tilde{a}_{c a}^{0} \\
\tilde{a}_{c b}^{0}
\end{array}\right) \text {. }
$$

The steady state density matrix elements are those for the two-level system already given in Eq. (4.3). According to Eq. (2.13) the spontaneous emission is proportional to $\rho_{\mathrm{cc}}^{0}$ which is identically zero. Therefore only the stimulated spectrum exists and is found to be

$$
\begin{aligned}
g_{\mathrm{st}}\left(\omega_{s}\right)= & \operatorname{Im}\left\{\left[\Delta_{b c}^{*}\left(\Delta_{a b} \Delta_{a b}^{*}+2 W^{2} \Gamma_{a b} / \Gamma_{b b}\right)\right.\right. \\
& \left.\left.-W^{2} \Delta_{a b}^{*}\right] D(0)^{-1} \cdot\left(\Delta_{a c}^{*} \Delta_{b c}^{*}-W^{2}\right)^{-1}\right\} .
\end{aligned}
$$

For the inhomogeneous distribution we make the same substitutions of Eq. (5.9) as in the previous section. The simulated absorption spectra are given in Figs. 13-15. In a homogeneous system the absorption line is Lorentzian in the weak field limit $W=0$, but broadens and finally splits into two line increasing Rabi frequency of the driving field (Fig. 13).

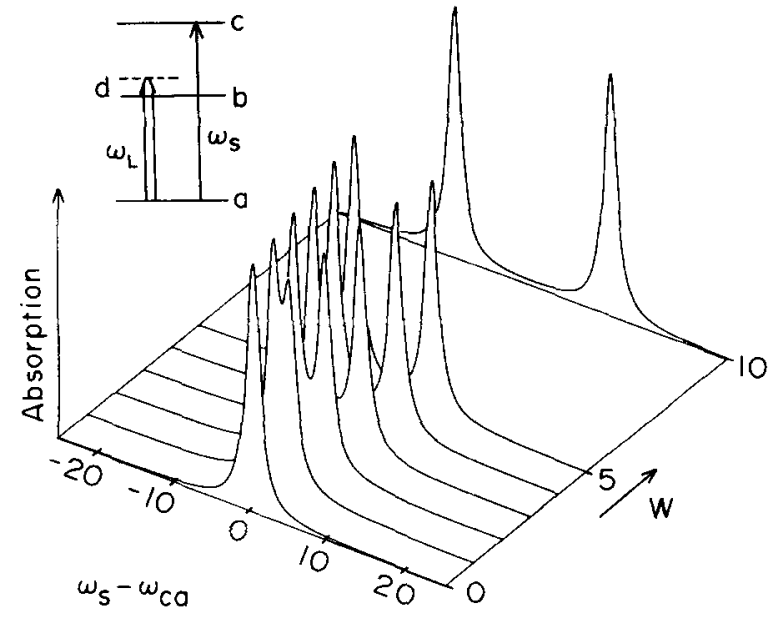

FIG. 13. Absorption spectrum of the $|a\rangle \rightarrow|c\rangle$ transition of a three level system with the $|a\rangle \rightarrow|b\rangle$ transition pumped by a strong field. The detuning is $d=\omega_{L}-\omega_{b a}=0.1$, the linewidth parameters are $\Gamma_{a b}=0.001$, $\Gamma_{b c}=1.001, \Gamma_{a c}=1, \Gamma_{b b}=0.002, \Gamma_{\mathrm{cc}}=2$ corresponding to no pure dephasing. A small inhomogeneous contribution of Gaussian shape is included with $\alpha=0.5, \sigma=0.5$.

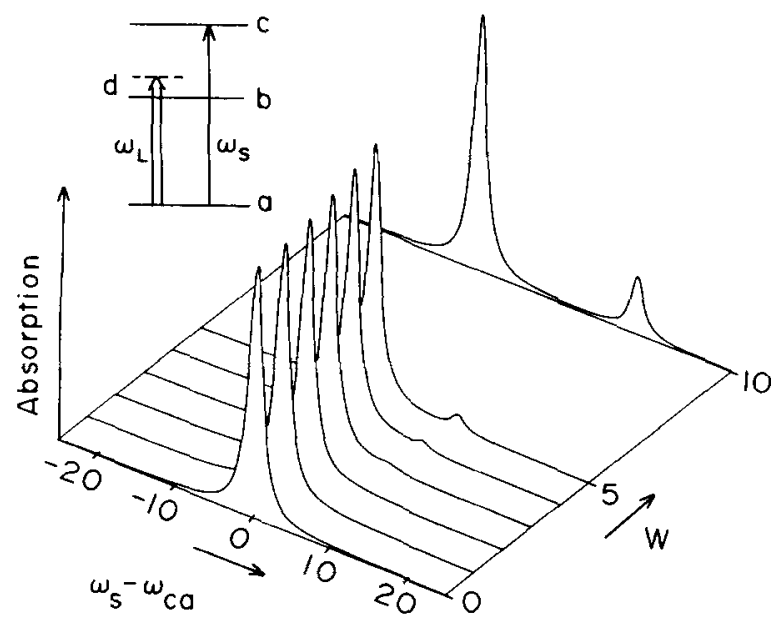

FIG. 14. Same as Fig. 13, but with the detuning set to $d=5$.

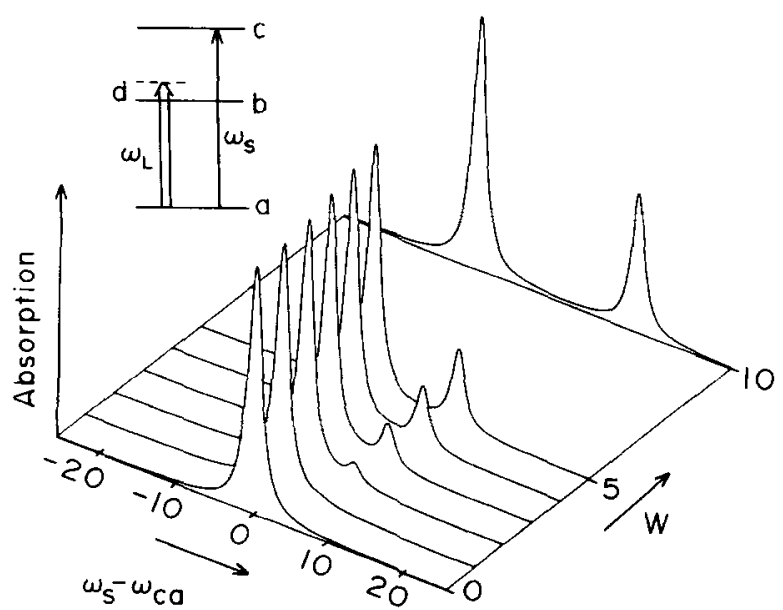

FIG. 15. Same as Fig. 14, but with a pure dephasing contribution $\Gamma^{\prime}=0.01$ added to all three linewidth parameters $\Gamma_{a b}, \Gamma_{a c}$, and $\Gamma_{b c}$. 


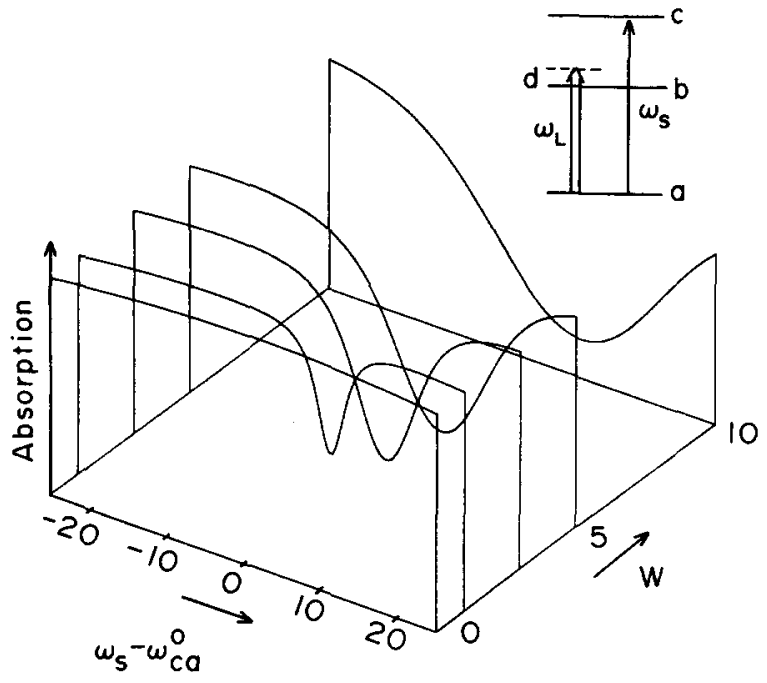

FIG. 16. Same as Fig. 14, but with the inhomogeneous distribution broadened to $\sigma=50$.

Adding a pure dephasing contribution of the same amount as in the fluorescence case in the previous section does not significantly alter these line shapes. With the driving field detuned the splitting of the line is unsymmetrical as shown in Fig. 14, and the sideband is significantly enhanced by the dephasing (Fig. 15).

Convolution with a broad inhomogeneous distribution presents a completely different line shape pattern: Without the driving field the absorption band just reproduces the broad inhomogeneous distribution function, in our case a Gaussian (Fig. 16). With the driving field switched on, a hole developed in this broad absorption line shape at $\omega_{s}-\omega_{c b}=(1-\alpha) d / \alpha$, where $d$ is the detuning of the driving field frequency from the center of the inhomogeneous distribution. With increasing $W$ this hole broadens, but does not split into two "negative lines" as occurred in the two level case (Fig. 9). The inclusion of pure dephasing contributions to the relaxation parameters results in significant additional broadening of this hole but no new features arise.

\section{SUMMARY}

In the present paper we have introduced a simple method to derive formulas and simulated line shapes for many types of noncoherent spectroscopies of systems under the influence of strong optical driving fields. Some of the results have been given before by other authors, but we believe the present approach to be particularly useful and generally applicable. In addition we give a general procedure to average all these spectra over inhomogeneously broadened distributions. Explicit formulas for Gaussian and Lorentzian distribution functions are given. This allows for modeling of fluorescence line narrowing, line narrowed resonance Raman, and non photochemical hole burning situations. The same procedure can however, be used to perform the ensemble average for coherent spectroscopies like CARS, CSRS, sum, and difference frequency generation in strongly driven systems, for which formulas of the homogeneous spectra have been given recently. ${ }^{1}$ The model can be further extended by adding rate equations connecting the populations to other levels not directly coupled to the light fields, thus incorporating photochemistry, ionization, or bottleneck bath states. ${ }^{44}$

'B. Dick and R. M. Hochstrasser, Chem. Phys. 75, 133 (1983).

${ }^{2}$ W. H. Louisell, Quantum Statistical Properties of Radiation (Wiley, New York, 1973), p. 360.

${ }^{3}$ S. Mukamel, Phys. Rep. 93, 1 (1982).

${ }^{4}$ B. R. Mollow, Phys. Rev. 188, 1969 (1969).

${ }^{3}$ B. R. Molloow, Phys. Rev. A 2, 76 (1970).

${ }^{6}$ B. R. Mollow, Phys. Rev. A 5, 2217 (1972).

'B. R. Mollow, Phys. Rev. A 12, 1919 (1975).

${ }^{8}$ B. R. Mollow, Phys. Rev. A. 5, 1522 (1972).

${ }^{9}$ B. R. Mollow, Phys. Rev. A. 15, 1023 (1977).

${ }^{10}$ S. Mukamel and A. Nitzan, J. Chem. Phys. 66, 2462 (1977).

${ }^{11}$ S. Mukamel, D. Grimbert, and Y. Rabin, Opt. Commun. 40, 185 (1982).

${ }^{12}$ S. Mukamel, D. Grimbert, and Y. Rabin Phys. Rev. A 26, 341 (1982).

${ }^{13}$ C. Cohen-Tannoudji, Lect. Notes 4 (Laser Spectroscopy) 325 (1975).

${ }^{14}$ C. Cohen-Tannoudji and S. Reynaud, J. Phys. B 10, 345 (1977).

${ }^{15}$ C. Cohen-Tannoudji and S. Reynaud, J. Phys. B 10, 365 (1977).

${ }^{16}$ A. Szöke and E. Courtens, Phys. Rev. Lett. 34, 1053 (1975).

${ }^{17}$ E. Courtens and A. Szöke, Phys. Rev. A 15, 1588 (1977).

${ }^{18}$ Y. Rabin and A. Ben-Reuven, J. Phys. B 13, 2011 (1980)

${ }^{19}$ Y. Rabin, D. Grimbert, and S. Mukamel, Phys. Rev. A 26, 271 (1982).

${ }^{20}$ J. Friedman and R. M. Hochstrasser, Chem. Phys. 6, 155 (1974).

${ }^{21}$ J. O. Berg, C. A. Langhoff, and G. W. Robinson, Chem. Phys. Lett. 29, 305 (1974).

${ }^{22}$ R. M. Hochstrasser and F. A. Novak, Chem. Phys. Lett. 41, 407 (1976).

${ }^{23}$ R. M. Hochstrasser and F. A. Novak, Chem. Phys. Lett. 53, 3 (1978).

${ }^{24}$ J. M. Friedman, F. Novak, and R. M. Hochstrasser, in Laser and Coherence Spectroscopy, edited by J. F. Steinfeld (Plenum, New York 1978), p. 451 .

${ }^{25}$ Y. R. Shen, Phys. Rev. B 9, 622 (1974).

${ }^{26}$ The rate of transitions between two subsystems is given through Fermi's golden rule by: $I=\int_{+\infty}^{-\infty} d t\langle V \cdot V(t)\rangle$, where $V$ is the interaction operator and the average involves the total density operator of the system in the absence of the interaction. For a two-level system and a field mode with creation and annihilation operators $b^{+}$and $b$ the interaction in the rotating wave approximation is

$$
V(t)=D \cdot\left(a_{v \mu}(t) b+b^{i \omega t}-a_{\mu v}(t) b e^{-i \omega t}\right)
$$

The average over the field density operator yields

$$
\begin{aligned}
I= & D^{2}(\bar{n}+1) \int_{-\infty}^{+\infty} d t\left\langle a_{\nu \mu} a_{\mu \nu}(t)\right\rangle e^{i \omega t} \\
& +D^{2} \bar{n} \int_{-\infty}^{+\infty} d t\left\langle a_{v \mu}(t) a_{\mu \nu}\right\rangle e^{i-\omega t}
\end{aligned}
$$

with no photons in the field initially $(\bar{n}=0)$ this gives the spontaneous result, while the terms proportional to $\bar{n}$ give the formula for stimulated processes. It can be brought into the form of Eq. (2.2) by substituting $t$ with $-t$ in the second integral and using the relation $\langle x|-t| y\rangle=\langle x y(t)\rangle$. The effect of the driving field is incorporated into the density operator of the molecular system semiclassically. The probe is described quantum mechanically through its operators $b$ and $b^{+}$.

${ }^{27}$ M. Lax, Phys. Rev. 129, 2342 (1963).

${ }^{28}$ M. Lax, Phys. Rev. 172, 350 (1968).

${ }^{29}$ S. H. Lin and H. Eyring, Proc. Natl. Acad. Sci. USA 74, 3105 (1977).

${ }^{30}$ S. H. Lin and H. Eyring, Proc. Natl. Acad. Sci. USA 74, 3623 (1977).

${ }^{31}$ We will distinguish the Laplace transform from the Fourier transform by using the superscript $L$ when confusion is prossible.

${ }^{32}$ See Ref. 1 for the numbering scheme for these transformation frequencies and a detailed discussion. For an $N$-level system $N-1$ transformation frequencies can be chosen independently, but in order to make the Liouville operator time independent, the transformation freqency for each level pair resonant with an applied field must be equal to that field frequency. Consequently, only one field can be applied to each level pair, and not more than $N-1$ fields to the whole system. We will deal in this paper with two-level and three-level systems subject to one applied field, whose frequency will be called $\omega_{L}$. When less than $N-1$ fields are applied, the other transformation frequencies can be regarded as parameters, and ac- 
cordingly identified with the frequency $\omega_{s}$ of the signal wave.

${ }^{33}$ Due to our definition of level ordering, $\mu>v$, we have $s_{\mu v}=1$ always.

${ }^{34}$ B. Dick and R. M. Hochstrasser, J. Chem. Phys. 78, 3398 (1983).

${ }^{35}$ J. Kikas and M. Rätsep, Phys. Status. Sol. B 112, 409 (1982).

${ }^{36}$ Handbook of Mathematical Functions, edited by M. Abramowitz and I.

A. Stegun, (Dover, New York, 1970), p. 295.

${ }^{37}$ The infinitesimal constant $\epsilon$ has been added to avoid a singularity at $v=0$ in the spontaneous spectrum. This divergence corresponds to the elastic Rayleigh scattering, and $\operatorname{Re}(1 / i v)$ is a representation of $\delta(v)$. The constant $\epsilon$ effectively transforms this $\delta$ function into a Lorentzian with width $2 \epsilon$, and a natural choice for $\epsilon$ would be the linewidth of the laser $\omega_{L}$. The divergence could alternatively be removed by treating elastic and inelastic scattering separately as in Ref. 5. Such a partitioning has the disadvantage that the expression for inelastic scattering has second order poles in the inhomogeneous distribution parameter $x$ making inhomogeneous ensemble averaging more complicated. ble averaging more complicated.

${ }^{38}$ M. D. Duncan, P. Oesterlin, F. Konig, and R. L. Byer, Chem. Phys. Lett. 80, 253 (1981).

${ }^{39}$ R. W. Boyd, M. G. Raymer, P. Narum, and D. J. Harter, Phys. Rev. A 24, 411 (1981).

${ }^{40}$ D. Block, R. K. Raj, K. S. Peng, and M. Ducloy, Phys. Rev. Lett. 49, 719 (1982).

${ }^{41}$ A. D. Wilson-Gordon, R. Klimovsky-Barid, and H. Friedmann, Phys. Rev. A 25, 1580 (1982).

${ }^{42}$ F. A. M. de Oliveira, C. B. de Arańjo, and J. R. Dios Leite, Phys. Rev. A 25, 2430 (1982).

${ }^{43}$ G. S. Agarwal and S. Singh, Phys. Rev. A 25, 3195 (1982).

${ }^{44}$ B. Dick and R. M. Hochstrasser, Chem. Phys. Lett. 102, 484 (1983).

${ }^{45}$ All spectra are symmetrical with respect to this detuning, i.e., replacing $d_{L}$ by $-d_{L}$ will yield the spectrum with the $d_{s}$ axis inverted. 